\title{
Atypical antipsychotics in the treatment of early-onset schizophrenia
}

This article was published in the following Dove Press journal:

Neuropsychiatric Disease and Treatment

I April 2015

Number of times this article has been viewed

\section{Michal Hrdlicka \\ Iva Dudova}

Department of Child Psychiatry, Charles University Second Faculty of Medicine and University Hospital Motol, Prague, Czech Republic
Correspondence: Michal Hrdlicka Department of Child Psychiatry, Charles University Second Faculty of Medicine, University Hospital Motol, V Uvalu 84, I 5006 Prague, Czech Republic

Tel +42022 4433400

Fax +420224433420

Email michal.hrdlicka@lfmotol.cuni.cz
Abstract: Atypical antipsychotics (AAPs) have been successfully used in early-onset schizophrenia (EOS). This review summarizes the randomized, double-blind, controlled studies of AAPs in EOS, including clozapine, risperidone, olanzapine, aripiprazole, paliperidone, quetiapine, and ziprasidone. No significant differences in efficacy between AAPs were found, with the exception of clozapine and ziprasidone. Clozapine demonstrated superior efficacy in treatment-resistant patients with EOS, whereas ziprasidone failed to demonstrate efficacy in the treatment of EOS. Our review also focuses on the onset of action and weight gain associated with AAPs. The data on onset of action of AAPs in pediatric psychiatry are scanty and inconsistent. Olanzapine appears to cause the most significant weight gain in patients with EOS, while ziprasidone and aripiprazole seem to cause the least.

Keywords: early-onset schizophrenia, atypical antipsychotics, efficacy, onset of action, weight gain

\section{Introduction}

Early-onset schizophrenia (EOS), defined by an onset before 18 years of age, has been mostly reported as a disorder with a poorer prognosis than adult-onset schizophrenia. According to a recent systematic review, only $15.4 \%$ of EOS patients experienced a "good" long-term outcome, while $24.5 \%$ experienced a "moderate" outcome and $60.1 \%$ a "poor" outcome. ${ }^{1}$ The prevalence of EOS has not been adequately studied. ${ }^{2}$ Some authors have reported that up to $20 \%$ of individuals with schizophrenia become ill prior to 18 years of age, ${ }^{3}$ which constitutes a significant minority that deserves special attention.

Atypical antipsychotics (AAPs) became an important part of modern psychiatric treatment during the 1990s, and significantly changed the treatment of schizophrenia by offering broader symptom control as well as a more favorable tolerability profile. ${ }^{4}$ AAPs have also been successfully used in EOS, and the US Food and Drug Administration has approved the use of aripiprazole, olanzapine, risperidone, quetiapine, and paliperidone in adolescents. ${ }^{5}$ However, the literature on their use in pediatric patients remains limited. There have been few randomized controlled trials (RCTs) in adolescent schizophrenia. ${ }^{6}$ The aim of our review is to summarize what we understand about the efficacy and safety of AAPs in EOS, with special attention paid to the onset of action of AAPs and the associated weight gain.

\section{Efficacy and safety}

Randomized, double-blind, controlled studies of AAPs in EOS are summarized in Table 1. The first five studies used head-to-head comparisons and three of them included clozapine and treatment-resistant patients. Starting in 2009, trials employing placebocontrolled comparisons prevailed. 
Table I Randomized, double-blind, controlled trials of atypical antipsychotics in early-onset schizophrenia (chronological order)

\begin{tabular}{|c|c|c|c|c|c|c|}
\hline Study & D (weeks) & TR & $\mathbf{N}$ & $\begin{array}{l}\text { Age } \\
\text { (years) }\end{array}$ & $\begin{array}{l}\text { Primary efficacy } \\
\text { measure }\end{array}$ & Efficacy \\
\hline Kumra et $\mathrm{al}^{7}$ & 6 & Yes & 21 & $14.0 \pm 2.3^{*}$ & BPRS & $\mathrm{CLO}>\mathrm{HAL}$ \\
\hline Sikich et al" & 8 & No & 50 & $8-19$ & BPRS-C & $\mathrm{RIS}=\mathrm{OLA}=\mathrm{HAL}$ \\
\hline Shaw et $\mathrm{a}^{8}$ & 8 & Yes & 25 & $7-16$ & SANS & $C L O \geq O L A$ \\
\hline Kumra et al ${ }^{9}$ & 12 & Yes & 39 & $10-18$ & BPRS & CLO $>$ OLA \\
\hline Sikich et $\mathrm{al}^{13}$ & 8 & No & 116 & $8-19$ & PANSS & $\mathrm{OLA}=\mathrm{RIS}=\mathrm{MOL}$ \\
\hline Findling et $\mathrm{al}^{15}$ & 6 & No & 302 & $10-17$ & PANSS & ARI $>$ pla \\
\hline Haas et $\mathrm{al}^{16}$ & 6 & No & 160 & $10-17$ & PANSS & RIS $>$ pla \\
\hline Kryzhanovskaya et al ${ }^{17}$ & 6 & No & 107 & $13-17$ & BPRS-C & OLA $>$ pla \\
\hline Singh et al ${ }^{18}$ & 6 & No & 201 & $12-17$ & PANSS & PAL $>$ pla \\
\hline Findling et $\mathrm{al}^{19}$ & 6 & No & 222 & $13-17$ & PANSS & QUE $>$ pla \\
\hline Findling et $\mathrm{al}^{5}$ & 6 & No & 283 & $13-17$ & BPRS-A & $\mathrm{ZIP}=\mathrm{pla}$ \\
\hline Savitz et al $^{14}$ & 8 & No & 228 & $12-17$ & PANSS & $P A L=A R I$ \\
\hline
\end{tabular}

Note: *Data shown are mean \pm standard deviation; the other data in the age column are shown as ranges.

Abbreviations: TR, treatment-resistance; D, duration; BPRS, Brief Psychiatric Rating Scale; BPRS-C, Brief Psychiatric Rating Scale for Children; SANS, Schedule for the Assessment of Negative Symptoms; PANSS, Positive and Negative Syndrome Scale; BPRS-A, Brief Psychiatric Rating Scale-Anchored; CLO, clozapine; HAL, haloperidol; RIS, risperidone; OLA, olanzapine; MOL, molindone; ARI, aripiprazole; pla, placebo; PAL, paliperidone extended release; QUE, quetiapine; ZIP, ziprasidone.

\section{Head-to-head comparisons}

Clozapine was initially studied in comparison with haloperidol. ${ }^{7}$ Clozapine was superior to haloperidol on all measures of psychosis and reduced both positive and negative symptoms. Clozapine produced more drowsiness and salivation, whereas haloperidol caused more insomnia. No cases of agranulocytosis were observed, but five patients developed neutropenia and three patients developed electroencephalographic abnormalities while on clozapine. One third of the clozapine group had to be discontinued due to adverse effects.

Another two trials compared clozapine with olanzapine. In a study by Shaw et $\mathrm{al}^{8}$ there were moderate to large differential treatment effects in favor of clozapine; however, these reached significance only for alleviation of negative symptoms from an antipsychotic-free baseline, as measured by the Schedule for the Assessment of Negative Symptoms. They observed an increased proportion of treatment-related adverse events and symptoms in the clozapine group. Clozapine was associated with significantly more hypertension and supine tachycardia and a near-significant increase in sustained enuresis. Two patients on clozapine and one patient on olanzapine had a transient drop in their absolute neutrophil count below 1,500 cells $/ \mu \mathrm{L}$. Kumra et $\mathrm{al}^{9}$ compared clozapine with "high-dose" olanzapine (up to $30 \mathrm{mg}$ /day). The primary efficacy measure was improvement, defined as a decrease of $30 \%$ or more in the total Brief Psychiatric Rating Scale (BPRS) score relative to baseline and a Clinical Global Impression Scale-Improvement rating of 1 or 2. Significantly, more clozapine-treated adolescents met the response criteria (66\%) than did olanzapine-treated patients (33\%). Five patients experienced a serious adverse event that led to premature termination of treatment, including one case of neutropenia that was surprisingly not in a clozapine-treated patient, but instead was in an olanzapinetreated patient.

Despite the fact that the three above-mentioned studies were performed in relatively small and obviously underpowered samples $(21,25$, and 39 patients), clozapine demonstrated significant superiority over active comparator antipsychotic compounds in trials of different lengths $(6,8$, and 12 weeks). It was rather unusual, and testified to the considerable magnitude of its effect. A systematic review of clozapine data in EOS by Schneider et al included not only RCTs but also open-label and retrospective studies. ${ }^{10}$ The authors also concluded that clozapine showed superior efficacy compared with other antipsychotics in the treatment of patients with refractory EOS.

The following two RCTs, which used head-to-head comparisons, compared drugs in groups of three. The first study was carried out in 50 children and adolescents with schizophrenia or other psychotic illnesses. ${ }^{11}$ At the endpoint, $74 \%$ of patients in the risperidone group, $88 \%$ in the olanzapine group, and 53\% in the haloperidol group had achieved significant improvement. Exploratory comparisons indicated that the magnitude of the antipsychotic response with atypical agents was comparable with that observed with haloperidol. Common side effects, ie, somnolence, extrapyramidal side effects, and weight gain, occurred in all three treatment groups. The second study by McClellan et al, employed olanzapine and risperidone (AAPs) and molindone (a typical antipsychotic drug). ${ }^{12}$ The primary 
outcome measure was response to treatment, defined as a Clinical Global Impression Scale-Improvement score of 1 or 2 and at least a $20 \%$ reduction in the total score from the Positive and Negative Syndrome Scale (PANSS). Several substantial study modifications were required to address safety, the use of adjunctive medications, and the termination of the olanzapine arm due to weight gain. ${ }^{12}$ No significant differences were found between treatment groups in response rates (molindone 50\%, olanzapine 34\%, and risperidone $46 \%$ ) or magnitude of symptom reduction. ${ }^{13}$ Adverse effects were frequent but differed among medications. Participants reported a variety of side effects: the molindone group reported significantly higher rates of drug-induced akathisia, the olanzapine group reported significantly higher rates of weight gain and increased appetite, and the risperidone group reported significantly higher rates of constipation. Adverse effects led to premature treatment termination in eight patients on molindone, six patients on olanzapine, and five patients on risperidone.

A recent head-to-head study by Savitz et al compared paliperidone extended-release (ER) in three flexible doses $(3,6$, or $9 \mathrm{mg} /$ day $)$ with aripiprazole in three flexible doses $\left(5,10\right.$, or $15 \mathrm{mg} /$ day). ${ }^{14}$ The primary efficacy measure was the mean change in PANSS total score. Responders were defined as those patients who achieved a $20 \%$ or greater reduction in the PANSS total score from baseline to endpoint. After 8 weeks of treatment, there was no significant difference between the groups in either the primary efficacy measure or in the responder rate $(69.7 \%$ versus $76.3 \%)$. The most common ( $>10 \%$ of patients) treatment-emergent adverse events for paliperidone ER were akathisia, headache, somnolence, tremor, and weight gain, and for aripiprazole, adverse events were worsening of schizophrenia and somnolence. The 8-week acute treatment study was followed by an 18-week double-blind maintenance period for a total trial duration of 26 weeks. At the endpoint on week 26, no significant differences between treatment groups emerged, but response rates increased slightly in both groups $(76.8 \%$ versus $81.6 \%$ ).

\section{Placebo-controlled studies}

Findling et al compared aripiprazole $10 \mathrm{mg}$ and $30 \mathrm{mg}$ daily with placebo in adolescent schizophrenia. ${ }^{15}$ Using the PANSS total score as the primary efficacy assessment, they found that both aripiprazole doses were superior compared with placebo in the acute treatment of EOS. Adverse events while taking aripiprazole that occurred in more than 5\% (of either aripiprazole group and with combined incidence at least twice that for placebo) were extrapyramidal disorders, somnolence, and tremor.

Haas et al examined flexible doses of risperidone 1-3 $\mathrm{mg}$ /day or risperidone 4-6 $\mathrm{mg}$ /day relative to a placebo control group. ${ }^{16}$ The primary efficacy measure was a baselineto-end point change in the PANSS total score. Significant improvement was found in both risperidone groups versus placebo. Generally, adverse events were more common in the risperidone groups (75\% and $76 \%$ ) versus placebo $(54 \%)$. More specifically, risperidone 4-6 mg/day compared with risperidone 1-3 mg/day had higher incidences of extrapyramidal disorder (39\% versus 33\%), dizziness (14\% versus $7 \%$ ), and hypertonia ( $14 \%$ versus $5 \%$ ). No prolactin-related adverse events occurred.

Kryzhanovskaya et al studied olanzapine versus placebo in adolescents with EOS. ${ }^{17}$ Compared with placebo-treated patients, olanzapine-treated adolescents had significant better results for the total score on the Brief Psychiatric Rating Scale for Children (BPRS-C), Clinical Global Impression ScaleSeverity of Illness (CGI-S), and the PANSS total and positive scores. A secondary measure was patient response rates, defined a priori as a $30 \%$ or greater reduction of the BPRS-C total score from baseline to endpoint and a CGI-S score of 3 or lower on the final assessment. The a priori-defined response rate did not differ between the olanzapine and placebo groups (37.5\% versus $25.7 \%$ ); additionally, extrapyramidal symptoms did not differ between the groups.

Singh et al performed a placebo-controlled comparison of paliperidone ER in three weight-based, fixed dosing (lowdose $1.5 \mathrm{mg} /$ day, medium-dose $3 \mathrm{mg}$ /day, and high-dose $6 \mathrm{mg}$ /day). ${ }^{18}$ The mean change in PANSS score from baseline to endpoint (primary efficacy variable) was significant for medium-dose paliperidone ER, but not for the low-dose or high-dose groups versus placebo. The responder rate (percentage of patients with $20 \%$ or more reduction in PANSS total score from baseline to endpoint) was significantly higher in the medium-dose (64.6\%) and high-dose (51.1\%) groups, but not in the low-dose group (38.9\%) versus placebo $(33.3 \%)$. Somnolence, akathisia, insomnia, headache, and tremors were reported as the most common $(>5 \%)$ adverse events associated with the paliperidone group.

In a different study by Findling et $\mathrm{al}^{19}$ adolescents with EOS were randomized to quetiapine (400 or $800 \mathrm{mg} /$ day) or placebo treatment groups. The primary efficacy measure was a change in the PANSS total score from baseline to day 42 . Quetiapine at both doses provided significant improvement relative to placebo, including the primary efficacy measure. The proportions of patients who achieved the a priori 
defined response criterion (ie, a $30 \%$ or more reduction in PANSS total score on day 42) were $38.4 \%, 36.5 \%$, and $26.0 \%$ in the quetiapine $400 \mathrm{mg} /$ day, quetiapine $800 \mathrm{mg} / \mathrm{day}$, and placebo groups, respectively, a difference that was not significant. The overall incidences of adverse events in the quetiapine $400 \mathrm{mg} /$ day, quetiapine $800 \mathrm{mg} /$ day, and placebo groups were $79.5 \%, 74.3 \%$, and $60.0 \%$, respectively, with medication-related adverse events reported in $56.2 \%$, $46.0 \%$, and $22.7 \%$ of cases, respectively. The most common adverse events associated with quetiapine were somnolence, headache, and dizziness.

In a third study by Findling et $\mathrm{al}^{5}$ subjects were randomized to flexible oral doses of ziprasidone (40-160 mg/day, based on weight) or placebo. The primary endpoint was a change from baseline on the Brief Psychiatric Rating ScaleAnchored (BPRS-A). A 6-week RCT was followed by a 26-week, open-label extension study. A planned interim analysis for the primary endpoint in the RCT resulted in early termination of both studies. Treatment of EOS with ziprasidone failed to differentiate itself from the placebo group; however, ziprasidone was generally well tolerated, with a neutral weight and metabolic profile. Somnolence and extrapyramidal disorders were the most common adverse events compared with the placebo group (19.7\% versus $6.7 \%$ and $11.4 \%$ versus $1.1 \%$, respectively).

\section{Onset of action}

The "onset of action" parameter also appears in the literature, where it is similarly referred to as the "speed of onset of action", "time to efficacy", or "time to first improvement". Onset of action was defined, for double-blind studies, as the time to the first statistically significant difference between active and placebo treatment groups, which could also be considered as the "time to onset" ${ }^{20}$ Some authors underscore the need for a three-arm design (active drug, comparative drug, and placebo), claiming that it is more accurate, ${ }^{21}$ while other methodologies have been found effective in retrospective studies. In retrospective studies, "onset of efficacy" is defined as the day when the first remark regarding "patient improvement" appeared in the patient's medical documentation.

In our review of the literature, focused on the onset of action of AAPs in the treatment of schizophrenia, we narrowed the list of studies to those that directly compared at least two AAPs. ${ }^{22}$ In total, we found twelve relevant studies for adult and adolescent schizophrenia and the results were surprisingly inconsistent. One half of the studies reported onset of action equivalency in AAPs, while the other half reported a faster onset of action for risperidone versus clozapine, olanzapine, and quetiapine. Additionally, a faster onset of action has been reported for ziprasidone versus aripiprazole and clozapine. ${ }^{22}$ The only pediatric study dealing with mean response time reported a faster onset of action for olanzapine (1.6 weeks) versus risperidone ( 2.3 weeks) and haloperidol (2.4 weeks). ${ }^{11}$

We performed a retrospective study in a group of 296 teenage patients treated for schizophrenic psychoses in our department between 1997 and 2007.22 The time to first improvement could be estimated in 258 patients; of these, $195(76 \%)$ had been treated with AAPs and 63 (24\%) with typical antipsychotics. The mean time to first improvement was 6.9 days in the AAP group and 5.8 days in the typical antipsychotic drug group; the difference was significant at the trend level $(P=0.063)$. With respect to individual drugs, the mean time to first improvement was 7.1 days for risperidone, 6.7 days for olanzapine, 6.5 days for ziprasidone, 6.1 days for quetiapine, 7.4 days for clozapine, 5.2 days for haloperidol, 5.9 days for perphenazine, and 6.0 days for sulpiride. Differences between the drugs were not significant.

\section{Weight gain}

AAPs were introduced to child and adolescent psychiatry in the belief that they were better tolerated, especially with regard to a lower risk of extrapyramidal side effects. ${ }^{6}$ This appeared to be true; however, other side effects soon became apparent. Among children and adolescents, weight gain, or an increase in body mass index, emerges as one of the most significant side effects of AAPs, with an ensuing risk of type 2 diabetes, cardiovascular morbidity, and metabolic syndrome. ${ }^{23}$

There are significantly more data available than just efficacy studies, therefore complete reliance on RCTs is not necessary. Fortunately, the data on weight gain obtained from open-label and retrospective studies are potentially as reliable and useful as data from RCTs. For example, if we compare data on olanzapine-associated weight gain derived from 6-week double-blind, open-label, and retrospective studies, ${ }^{17,24,25}$ we see very similar results $(4.3 \mathrm{~kg}, 4.6 \mathrm{~kg}$, and $4.4 \mathrm{~kg}$, respectively).

Several recent comprehensive reviews have concluded that AAPs, compared with typical antipsychotic drugs, are associated with a greater risk for antipsychotic-induced weight gain, and among the AAPs, olanzapine appears to cause the most significant weight gain, while ziprasidone seems to cause the least. ${ }^{6,23}$

It is important to compare the dynamics of weight gain between short-term and long-term studies. Unfortunately, 
both short-term and long-term data are available only for some antipsychotics, and this narrowed our comparison (Table 2). In our short-term, retrospective EOS study, adolescents gained an average of $3.6 \mathrm{~kg}$ on risperidone, $4.4 \mathrm{~kg}$ on olanzapine, and $2.1 \mathrm{~kg}$ on clozapine during a 6-week treatment period. ${ }^{25}$ These results are very similar to those described in a 6-week, open-label study by Fleischhaker et al who reported an average weight gain of $2.8 \mathrm{~kg}$ for risperidone, $4.6 \mathrm{~kg}$ for olanzapine, and $2.5 \mathrm{~kg}$ for clozapine. ${ }^{24}$ Weight gain reported from other 6-week studies include: quetiapine $400 \mathrm{mg}$ /day, $2.2 \mathrm{~kg}$ versus quetiapine $800 \mathrm{mg} /$ day, $1.8 \mathrm{~kg} ;{ }^{19}$ aripiprazole $10 \mathrm{mg} /$ day, $0.0 \mathrm{~kg}$ versus aripiprazole $30 \mathrm{mg} /$ day, $0.2 \mathrm{~kg} ;{ }^{15}$ and ziprasidone $-0.1 \mathrm{~kg} .{ }^{5}$ Results for paliperidone ER are as follows: $0.3 \mathrm{~kg}$ for low-dose, $1.1 \mathrm{~kg}$ for mediumdose, and $1.4 \mathrm{~kg}$ for high-dose treatment groups. ${ }^{18} \mathrm{~A} 3$-month cohort study of first-time use of AAPs in children and adolescents reported a mean weight gain of $8.5 \mathrm{~kg}$ with olanzapine, $6.1 \mathrm{~kg}$ with quetiapine, $5.3 \mathrm{~kg}$ with risperidone, and $4.4 \mathrm{~kg}$ with aripiprazole. ${ }^{26}$ A 6-month cohort study in drug-naive patients found a mean weight gain of $11.5 \mathrm{~kg}$ with olanzapine, $7.1 \mathrm{~kg}$ with risperidone, and $6.3 \mathrm{~kg}$ with quetiapine. ${ }^{27}$ Finally, a 6-month RCT reported a mean weight gain of $2.3 \mathrm{~kg}$ for paliperidone ER and $0.40 \mathrm{~kg}$ for aripiprazole. ${ }^{14}$

A rough analysis of these results shows that the weight gain continually increased with AAPs until the third month, after which it either plateaued (quetiapine, $6.1 \mathrm{~kg}$ versus $6.3 \mathrm{~kg}$ in 3-month and 6-month comparisons), or the speed of weight gain substantially diminished (risperidone, $5.3 \mathrm{~kg}$ versus $7.1 \mathrm{~kg}$ in 3-month and 6-month comparisons). However, this trend was less apparent for olanzapine $(8.5 \mathrm{~kg}$ versus $11.5 \mathrm{~kg}$ in 3-month and 6-month comparisons). Thus, the observations support the recommendation of the American Academy of Child and Adolescent Psychiatry that the risk of weight gain may limit the use of olanzapine as a first-line agent. ${ }^{2}$

\section{Discussion}

Published RCTs in EOS differed substantially in sample size and methodology, and this made comparison and generalization somewhat difficult. Initial studies included at most 50 patients, ${ }^{7-9,11}$ ie, subject numbers that would have been barely acceptable in adult psychiatry due to an underpowered design. The situation only improved in 2008 when the first larger and better designed studies appeared; a remarkable and noteworthy delay compared with adult psychiatry. Most studies lasted 6 weeks, but some lasted 8 weeks, and one study in treatment-resistant patients lasted 12 weeks (Table 1). Six studies used the PANSS as the primary scale for efficacy assessment, five studies used the BPRS and its modifications (BPRS-C, BPRS-A), and one study used the Schedule for the Assessment of Negative Symptoms (Table 1). Some trials had no a priori definition of response and some did (although, in different ways), such as a reduction in the BPRS or PANSS total scores by $30 \%$ or more ${ }^{9,17,19}$ or a reduction in the PANSS total score by $20 \%$ or more. ${ }^{13,14,18}$ These a priori-defined responses of $20 \%-30 \%$, from a clinical perspective, represent relatively small changes; however, at the same time we must take into account that AAPs are drugs with calculated effect sizes in the range of $0.11-0.29$, which according to Cohen's classification would be small to moderate. ${ }^{28}$

We have concluded, along with many other authors, that there are no significant differences in efficacy between AAPs, with the exception of clozapine and ziprasidone. ${ }^{5,6,10}$ Clozapine demonstrated superior efficacy in treatment-resistant patients with $\operatorname{EOS}^{6,10}$ and probably would demonstrate such efficacy in the general EOS population if its use, due to hematological side effects, were not limited to refractory patients. The position of ziprasidone for treatment of EOS, on the other hand, has been weakened by negative results from a pediatric $\mathrm{RCT},{ }^{5}$ and according to the American Academy of Child and Adolescent

Table 2 Weight gain associated with selected atypical antipsychotics: short-term and long-term studies

\begin{tabular}{|c|c|c|c|c|c|c|c|}
\hline \multirow[t]{2}{*}{ Study } & \multirow[t]{2}{*}{ Duration } & \multirow[t]{2}{*}{ Design } & \multicolumn{5}{|c|}{ Weight gain with AAPs (kg) } \\
\hline & & & RIS & OLA & QUE & PAL & ARI \\
\hline Fleischhaker et a ${ }^{24}$ & 6 weeks & O & 2.8 & 4.6 & & & \\
\hline Hrdlicka et $\mathrm{al}^{25}$ & 6 weeks & $\mathrm{R}$ & 3.6 & 4.4 & & & \\
\hline Findling et al ${ }^{19}$ & 6 weeks & RCT & & & 1.8 & & \\
\hline Findling et $\mathrm{al}^{15}$ & 6 weeks & RCT & & & & & 0.2 \\
\hline Singh et al ${ }^{18}$ & 6 weeks & RCT & & & & 1.4 & \\
\hline Correll et $\mathrm{a}^{26}$ & 3 months & O & 5.3 & 8.5 & 6.1 & & 4.4 \\
\hline Arango et $\mathrm{a}^{27}$ & 6 months & O & 7.1 & 11.5 & 6.3 & & \\
\hline Savitz et al ${ }^{14}$ & 6 months & RCT & & & & 2.3 & 0.4 \\
\hline
\end{tabular}

Abbreviations: AAPs, atypical antipsychotics; O, open-label study; R, retrospective study; RCT, randomized, controlled trial; RIS, risperidone; OLA, olanzapine; QUE, quetiapine; PAL, paliperidone extended-release; ARI, aripiprazole. 
Psychiatry, it should not be considered for EOS, ${ }^{2}$ even though its efficacy in adult schizophrenia is beyond doubt. ${ }^{29-31}$

Generally speaking, we found limited data on the efficacy of AAPs in the treatment of EOS; however, we found a definitive lack of efficacy data with regard to specific subtypes of schizophrenia, particularly catatonic schizophrenia. Experience from adult psychiatry shows that typical antipsychotic drugs may worsen catatonic symptoms and should be avoided if symptoms of catatonia are already present. ${ }^{32}$ There are some studies and case reports in adult populations demonstrating the benefits of AAPs such as clozapine, olanzapine, risperidone, and quetiapine in catatonia. ${ }^{33}$ In pediatric psychiatry, only case reports for olanzapine, ${ }^{34,35}$ aripiprazole, ${ }^{36,37}$ and risperidone ${ }^{38}$ are available.

This review also demonstrates that the amount of data regarding the speed of onset of action of AAPs in pediatric psychiatry leaves much to be desired, and future study designs that feature head-to-head comparisons would be extremely valuable. With regard to weight gain, lack of data is not an issue. Since the meta-analysis by Allison et al first highlighted the topic, ${ }^{39}$ and right through to the present, an abundance of data has been generated concerning use of AAPs and weight gain in both adult and pediatric psychiatry. Unfortunately, ziprasidone, an atypical antipsychotic drug that causes the least weight gain, also appears to be ineffective in EOS. ${ }^{5}$ Among the effective treatments for EOS, aripiprazole appears to cause the least weight gain. ${ }^{14}$

Our review confirms a shortage of RCTs in EOS, and also reveals a decreasing trend in publishing head-to-head RCTs. Since 2008, only one head-to-head RCT has been published. ${ }^{14}$ This might be associated with the disappointment arising from the premature termination of the olanzapine arm in the McClellan et al study (due to weight gain), which skewed the results of the entire study. ${ }^{12}$ In contrast, numerous comparative head-to-head studies have enabled extensive meta-analyses that have provided important information concerning the treatment of schizophrenia in adult populations, ${ }^{28}$ something that is lacking in the fields of child and adolescent psychiatry. Considering the timeline, the majority of RCTs was published in a 6-year period between 2004 and 2009 (seven RCTs), whereas only four RCTs were published subsequently (2010 to present). This may reflect stagnation in the extension of experience from adult psychiatry to pediatric psychiatry, stagnation in development of new atypical antipsychotic compounds (in general), or a particular new trend, expressed in the words of Sarah Webb:
Whereas the pharmaceutical industry has been increasingly shying away from psychiatric disorders, such as schizophrenia and depression, interest in autism has intensified..$^{40}$

\section{Acknowledgments}

This research was supported by the Conceptual Development of Research Organization (University Hospital Motol, Prague, Czech Republic; 00064203) and by the Programme for the Development of Fields of Study at Charles University (P07, Psychosocial Aspects of the Quality of Human Life). The authors would like to thank Thomas Secrest for his assistance with the English version of the manuscript.

\section{Disclosure}

$\mathrm{MH}$ has been a speaker at events sponsored by Eli Lilly in the Czech Republic. ID has no conflict of interest to report.

\section{References}

1. Clemmensen L, Lammers Vernal D, Steinhausen HC. A systematic review of the long-term outcome of early onset schizophrenia. BMC Psychiatry. 2012;12:150.

2. McClellan J, Stock S; American Academy of Child and Adolescent Psychiatry (AACAP) Committee on Quality Issues (CQI). Practice parameter for the assessment and treatment of children and adolescents with schizophrenia. J Am Acad Child Adolesc Psychiatry. 2013;52:976-990.

3. Sadock BJ, Sadock VA, Ruiz P, editors. Kaplan and Sadock's Comprehensive Textbook of Psychiatry. 9th ed. Philadelphia, PA, USA: Lippincott Williams \& Wilkins; 2009.

4. Fleischhacker WW, Hummer M. Drug treatment of schizophrenia in the 1990s. Achievements and future possibilities in optimising outcomes. Drugs. 1997;53:915-929.

5. Findling RL, Cavus I, Pappadopulos E, et al. Ziprasidone in adolescents with schizophrenia: results from a placebo-controlled efficacy and long-term open-extension study. J Child Adolesc Psychopharmacol. 2013;23:531-544

6. Fraguas D, Correll CU, Merchan-Naranjo J, et al. Efficacy and safety of second-generation antipsychotics in children and adolescents with psychotic and bipolar spectrum disorders: comprehensive review of prospective head-to-head and placebo-controlled comparisons. Eur Neuropsychopharmacol. 2011;21:621-645.

7. Kumra S, Frazier JA, Jacobsen LK, et al. Childhood-onset schizophrenia: a double-blind clozapine-haloperidol comparison. Arch Gen Psychiatry. 1996;53:1090-1097.

8. Shaw P, Sporn A, Gogtay N, et al. Childhood-onset schizophrenia: a double-blind, randomized clozapine-olanzapine comparison. Arch Gen Psychiatry. 2006;63:721-730.

9. Kumra S, Kranzler H, Gerbino-Rosen G, et al. Clozapine and "high-dose" olanzapine in refractory early-onset schizophrenia: a 12-week randomized and double-blind comparison. Biol Psychiatry. 2008;63:524-529.

10. Schneider C, Corrigan R, Hayes D, Kyriakopoulos M, Frangou S. Systematic review of the efficacy and tolerability of clozapine in the treatment of youth with early onset schizophrenia. Eur Psychiatry. 2014;29:1-10.

11. Sikich L, Hamer RM, Bashford RA, Sheitman BB, Lieberman JA. A pilot study of risperidone, olanzapine, and haloperidol in psychotic youth: a double-blind, randomized, 8-week trial. Neuropsychopharmacology. 2004;29:133-145. 
12. McClellan J, Sikich L, Findling RL, et al. Treatment of Early-Onset Schizophrenia Spectrum Disorders (TEOSS): rationale, design, and methods. J Am Acad Child Adolesc Psychiatry. 2007;46:969-978.

13. Sikich L, Frazier JA, McClellan J, et al. Double-blind comparison of first- and second-generation antipsychotics in early-onset schizophrenia and schizoaffective disorder: findings from the treatment of early-onset schizophrenia spectrum disorders (TEOSS) study. Am J Psychiatry. 2008;165:1420-1431.

14. Savitz AJ, Lane R, Nuamah I, Gopal S, Hough D. Efficacy and safety of paliperidone extended release in adolescents with schizophrenia: a randomized, double-blind study. J Am Acad Child Adolesc Psychiatry. 2015;54:126-137.

15. Findling RL, Robb A, Nyilas M, et al. A multiple-center, randomized, double-blind, placebo-controlled study of oral aripiprazole for treatment of adolescents with schizophrenia. Am J Psychiatry. 2008;165:1432-1441.

16. Haas M, Unis AS, Armenteros J, Copenhaver MD, Quiroz JA, Kushner SF. A 6-week, randomized, double-blind, placebo-controlled study of the efficacy and safety of risperidone in adolescents with schizophrenia. J Child Adolesc Psychopharmacol. 2009;19:611-621.

17. Kryzhanovskaya L, Schultz C, McDougle C, et al. Olanzapine versus placebo in adolescents with schizophrenia: a 6-week, randomized, double-blind, placebo-controlled trial. J Am Acad Child Adolesc Psychiatry. 2009;48:60-70.

18. Singh J, Robb A, Vijapurkar U, Nuamah I, Hough D. A randomized, double-blind study of paliperidone extended-release in treatment of acute schizophrenia in adolescents. Biol Psychiatry. 2011;70:1179-1187.

19. Findling RL, McKenna K, Earley WR, Stankowski J, Pathak S. Efficacy and safety of quetiapine in adolescents with schizophrenia investigated in a 6-week, double-blind, placebo-controlled trial. J Child Adolesc Psychopharmacol. 2012;22:327-342.

20. Thase ME. Methodology to measure onset of action. J Clin Psychiatry. 2001;62 Suppl 15:18-21.

21. Thompson C. Onset of action of antidepressants: results of different analyses. Hum Psychopharmacol Clin Exp. 2002;17:S27-S32.

22. Zedkova I, Dudova I, Urbanek T, Hrdlicka M. Onset of action of atypical and typical antipsychotics in the treatment of adolescent schizophrenic psychoses. Neuroendocrinol Lett. 2011;32:667-670.

23. Martinez-Ortega JM, Funes-Gogoy S, Diaz-Atienza F, Gutierrez-Rojas L, Perez-Costillas L, Gurpegui M. Weight gain and increase of body mass index among children and adolescents treated with antipsychotics: a critical review. Eur Child Adolesc Psychiatry. 2013;22:457-479.

24. Fleischhaker C, Heiser P, Hennighausen K, et al. Weight gain associated with clozapine, olanzapine and risperidone in children and adolescents. J Neural Transm. 2007;114:273-280.

25. Hrdlicka M, Zedkova I, Blatny M, Urbanek T. Weight gain associated with atypical and typical antipsychotics during treatment of adolescent schizophrenic psychoses: a retrospective study. Neuroendocrinol Lett. 2009;30:256-261.
26. Correll CU, Manu P, Olshanskiy V, Napolitano B, Kane JM, Malhotra AK Cardiometabolic risk of second-generation antipsychotic medications during first-time use in children and adolescents. JAMA. 2009;302:1765-1773.

27. Arango C, Giraldez M, Merchan-Naranjo J, et al. Second-generation antipsychotic use in children and adolescents: a six-month prospective, cohort study in drug-naive patients. J Am Acad Child Adolesc Psychiatry. 2014;53:1179-1190.

28. Leucht S, Komossa A, Rummel-Kluge C, et al. A meta-analysis of head-to-head comparisons of second-generation antipsychotics in the treatment of schizophrenia. Am J Psychiatry. 2009;166:152-163.

29. Simpson GM, Glick ID, Weiden PJ, Romano SJ, Siu CO. Randomized, controlled, double-blind multicenter comparison of the efficacy and tolerability of ziprasidone and olanzapine in acutely ill inpatients with schizophrenia or schizoaffective disorder. Am J Psychiatry. 2004;161:1837-1847.

30. Rosenheck RA, Leslie DL, Sindelar J, et al. Cost-effectiveness of second-generation antipsychotics and perphenazine in a randomized trial of treatment for chronic schizophrenia. Am J Psychiatry. 2006;163:2080-2089.

31. Sacchetti E, Galluzzo A, Valsecchi P, Romeo F, Gorini B, Warrington L. Ziprasidone vs clozapine in schizophrenia patients refractory to multiple antipsychotic treatments: the Mozart study. Schizophr Res. 2009;110:80-89.

32. Blumer D. Catatonia and the neuroleptics: psychobiologic significance of remote and recent findings. Compr Psychiatry. 1997;38:193-201.

33. Sienaert P, Dhossche DM, Vancampfort D, De Hert M, Gazdag G. A clinical review of the treatment of catatonia. Front Psychiatry. 2014;5:181.

34. DelBello MP, Foster KD, Strakowski SM. Case report: treatment of catatonia in an adolescent male. $J$ Adolesc Health. 2000;27:69-71.

35. Dudova I, Hrdlicka M. Successful use of olanzapine in adolescent monozygotic twins with catatonic schizophrenia resistant to electroconvulsive therapy. Neuroendocrinol Lett. 2008;29:47-50.

36. Strawn JR, Delgado SV. Successful treatment of catatonia with aripiprazole in an adolescent with psychosis. JChild Adolesc Psychopharmacol. 2007; 17:733-735.

37. Kirino E. Prolonged catatonic stupor successfully treated with aripiprazole in an adolescent male with schizophrenia: a case report. Clin Schizophr Relat Psychoses. 2010;4:185-188.

38. Paraga H, Harris K. Catatonia under treatment with risperidone. J Dev Behav Pediatr. 2006;27:369.

39. Allison DB, Mentore JL, Heo M, et al. Antipsychotic-induced weight gain: a comprehensive research synthesis. Am J Psychiatry. 1999;156: $1688-1696$

40. Webb S. Drugmakers dance with autism. Nat Biotechnol. 2010;28 $772-774$.
Neuropsychiatric Disease and Treatment

\section{Publish your work in this journal}

Neuropsychiatric Disease and Treatment is an international, peerreviewed journal of clinical therapeutics and pharmacology focusing on concise rapid reporting of clinical or pre-clinical studies on a range of neuropsychiatric and neurological disorders. This journa is indexed on PubMed Central, the 'PsycINFO' database and CAS,

\section{Dovepress}

and is the official journal of The International Neuropsychiatric Association (INA). The manuscript management system is completely online and includes a very quick and fair peer-review system, which is all easy to use. Visit http://www.dovepress.com/testimonials.php to read real quotes from published authors. 